\title{
Apolipoprotein B Is a Good Tool for Screening Dyslipidaemia in Apparently Healthy Population
}

\author{
L. V. Athiththan*, M. F. F. Nusha, U. P. K. Hettiaratchi, P. P. R. Perera \\ Department of Biochemistry, University of Sri Jayewardenepura, Nugegoda, Sri Lanka \\ Email: "lohinia@sjp.ac.lk, farooknusha@gmail.com, usha@sjp.ac.lk, rasika@sjp.ac.lk
}

Received 12 April 2016; accepted 16 May 2016; published 19 May 2016

Copyright (C) 2016 by authors and Scientific Research Publishing Inc.

This work is licensed under the Creative Commons Attribution International License (CC BY).

http://creativecommons.org/licenses/by/4.0/

(c) (i) Open Access

\begin{abstract}
Dyslipidaemia is the major risk factor for cardiovascular disease (CVD) which is the leading cause of death in the world. Even though several lipid parameters are used, currently apolipoprotein B (apoB) is considered as the best predictor of CVD. Thus this study was carried out to find out the association between conventional lipid parameters and apoB in apparently healthy subjects. $A$ descriptive cross-sectional study was carried out in 170 apparently healthy volunteers who were not diagnosed with dyslipidaemia. After 12 hours overnight fast venous blood was obtained and Total cholesterol (TC), Triglyceride (TG), High density lipoprotein cholesterol (HDL-C) were measured by enzymatic kit method. Low density lipoprotein cholesterol (LDL-C) was calculated by Friedewald formula and apoB was analyzed by immune turbid metry using a Konelab ${ }^{\circledR}$ auto analyzer. Among the participants, majority $(63.5 \%)$ were females. The mean value of apoB concentration of the population was $103 \pm 42 \mathrm{mg} / \mathrm{dL}$ which was similar and not significantly different between the genders (Males, $102 \pm 37 \mathrm{mg} / \mathrm{dL}$ and Females, $104 \pm 45 \mathrm{mg} / \mathrm{dL}$ ). All lipid parameters showed a positive correlation with apoB concentration whereas HDL-C had a negative correlation $(r=-0.165)$. HDL-C significantly $(p<0.05)$ decreased with increase in apoB concentration while LDL-C, TC/HDL-C and non-HDL-C significantly $(p<0.05)$ increased with an increase in apoB concentration. Present study suggests that serum apoB has better correlations and associations with the parameters that are used in conventional lipid profile and with markers recommended for diagnosing dyslipidaemia. Hence apoB could be used as a single marker for screening dyslipidaemia in apparently healthy people.
\end{abstract}

\section{Keywords}

Apolipoprotein B, Dyslipidaemia, High Density Lipoprotein, Low Density Lipoprotein

\footnotetext{
"Corresponding author.
}

How to cite this paper: Athiththan, L.V., Nusha, M.F.F., Hettiaratchi, U.P.K. and Perera, P.P.R. (2016) Apolipoprotein B Is a Good Tool for Screening Dyslipidaemia in Apparently Healthy Population. Journal of Biosciences and Medicines, 4, 58-64. 


\section{Introduction}

Cardiovascular diseases (CVD) are the leading cause of mortality in the world [1]. According to the Ministry of Health, Sri Lanka it is the major cause of death in Sri Lankan hospitals as well [2]. Major risk factors associated with CVD are dyslipidaemia, hypertension, dysglycaemia and obesity where dyslipidaemia is found to be the most common risk factor [3]. CVD could be controlled by addressing these risk factors and taking proper preventive methods.

Dyslipidaemia is defined as elevated levels of total cholesterol (TC), low density lipoprotein cholesterol (LDL-C) and/or triglycerides (TG) with low level of high density lipoprotein cholesterol (HDL-C) compared with the recommended cut-off values, which is collectively known as atherogenic dyslipidaemia [4]. It is found that South Asians who live round the world have higher incidence of CVD which is associated with a higher prevalence of dyslipidaemia [5]. It has also been reported that the prevalence of dyslipidaemia in Sri Lanka was very high where hypercholesterolemia, hypertriglyceridaemia, high LDL-C and low HDL-C were 53.6\%, 22.7\%, $24.7 \%$ and $53.1 \%$ respectively [6] indicating the need for proper screening, diagnosis, preventive and control methods.

The latest lipid lowering guidelines recommended by the National Cholesterol Education Programme (NCEP), Adult Treatment Panel (ATP) III for atherogenic dyslipidaemia are primarily targeted on reducing LDL-C which is the predominant atherogenic particle [7]. Even though LDL-C is recommended as a marker for atherogenic dyslipidaemia, it does not accurately reflect in patients with hypertriglyceridaemia and diabetes mellitus.

In addition, the NCEP (ATP III) guidelines also add non-HDL-C as a secondarily targeted lipid parameter in individuals with hypertriglyceridaemia. Other study findings also indicate that, triglyceride (TG) rich lipoproteins such as very low density lipoprotein cholesterol (VLDL-C) and intermediate density lipoprotein cholesterol (IDL-C) have atherogenic effects in addition to small dense LDL-C [8]. Non-HDL-C which represents all the atherogenic lipid parameters of the sample is commonly used to determine the risk of CVD [9].

As small dense LDL-C has low affinity for LDL receptor, it is more susceptible to undergo oxidative modification, hence easily penetrating the arterial wall and exerting high atherogenic effects compared to other lipid parameters [8].

Apolipoprotein B (apoB) is the structural protein of all of these atherogenic lipoproteins where each molecule of VLDL-C, IDL-C, LDL-C and small dense LDL-C consists of one molecule of apoB. Thus, plasma concentration of apoB indicates the total number of atherogenic lipid particles providing more holistic information that could not be obtained from a traditional lipid profile. Lipoproteins are modified by glycaemic oxidation and apoB is more stable than the lipoproteins even in hyperglycaemic condition. Therefore, apoB is a good indicator of dyslipidaemia in diabetic patients [10]. Further, apoB level is not affected by prandial status of an individual. Therefore, measurement of apoB does not require a fasting specimen for analysis [10].

Several studies have proven that $\mathrm{apoB}$ is a strong predictor to assess the risk of CVD and a good marker for dyslipidaemia with higher sensitivity and specificity as a predictive variable in both men and women irrespective of age [11]. Queback cardiovascular study suggests that apoB is an important marker in the assessment of heart disease especially in males [12]. Further, apoB is used as a biochemical marker instead of the conventional lipid profile and it is incorporated as a marker in the lipid profile worldwide. However in Sri Lanka, use of apoB as a marker is limited and it is not commonly used in the clinical practice.

The prevalence and pattern of dyslipidaemia vary among different ethnicities and it is influenced by genetic and acquired factors [13]. ApoB varies within the population due to genetic variations as a result of the existence of many different hyper variable regions at a single locus within a population [14]. Therefore, it is important to have specific values for a given population.

Even though studies related to apoB measurements have been carried out, most of the study subjects in those studies were on either lipid lowering therapy, or CVD patients. There is no documented evidence on apoB levels among healthy adults in Sri Lanka. Therefore, this study was carried out to assess the association between conventional lipid parameters which are used to measure dyslipidaemia and apoB to find out whether apoB can be used as a single biochemical marker for the screening and diagnosis of dyslipidaemia in apparently healthy adults.

\section{Methods}

\subsection{Study Design and Sampling}

This descriptive cross-sectional study was carried out at the Family Practice Centre and the Department of Bio- 
chemistry, Faculty of Medical Sciences, University of Sri Jayewardenepura, Sri Lanka. The study protocol was approved by the Ethics Review Committee of the Faculty of Medical Sciences, University of Sri Jayewardenepura. Sample size was calculated based on the equation $\left(\mathrm{n}=\left(\mathrm{z}^{2} * \sigma^{2}\right) / \mathrm{d}^{2}\right)$ where $\mathrm{z}$ is the significance level (1.96), $\sigma$ is the standard deviation and $\mathrm{d}$ is the precision. Informed written consent was obtained from all 170 volunteer participants prior to the study. Non probability, convenience sampling technique was used to recruit study participants during February 2013 to October 2013. Non diabetic adults aged between 20 to 70 years and without a past history of myocardial infarction, cancer and heart failure were included whereas hypercholesterolaemic, hypertensive, pregnant women and patients with cognitive impairments were excluded.

\subsection{Data Collection}

A questionnaire was administered by the interviewer to gather socio-demographic data [age, gender, area of residence, ethnicity, occupation and education level (categorized according to the Sri Lankan education system)] from all the participants.

\subsection{Biochemical Analysis}

After 12 hour overnight fasting, $3 \mathrm{~mL}$ of venous blood was obtained from all the participants adhering to universal precautions and standard protocols. Serum was separated and analysed for TC, TG and HDL-C by commercially available enzymatic kit methods (Stanbio ${ }^{\circledR}$ kits) based on Beer-Lambert's law on the same day of sample collection. An aliquot of serum sample was stored at $-20^{\circ} \mathrm{C}$ and batch analysis of apoB was done on a Konelab $^{\circledR}$ auto analyser based on the immunoturbidimetry method. Following ratios were obtained: TC/HDL-C, LDL-C/HDL-C and TG/HDL-C and LDL-C was calculated using the Fried-Wald equation [LDC-C = TC (HDL-C) - TG/5]. Non-HDL-C was calculated by subtracting HDL-C from TC [Non-HDL-C = (TC - HDL-C)].

The population was categorized based on the lipid parameters according to the cut off values recommended by the National Cholesterol Education Program (NCEP) Adult Treatment Panel III (ATP III) guidelines.

\subsection{Statistical Analysis}

All data were analysed using the Statistical Package for Social Science (SPSS) software version 17.0. Continuous variables are presented as mean \pm standard deviation (SD) and categorical variables are presented as percentages. Pearson correlation and Independent Sample t-tests were done to assess the correlation and association between conventional lipid parameters and apoB.

\section{Results}

\subsection{Socio-Demographic Data of the Study Subjects}

Among the participants, more than half of the population were females (63.5\%) with majority of them being less than 50 years of age, with educational level secondary or above and residing in urban or sub-urban areas (Table $1)$.

\subsection{Mean Values of the Lipid Parameters Assessed}

Even though mean values of most of the lipid parameters, TC, HDL-C, TG, TC/HDL-C and LDL-C/HDL-C were within the optimal cut off values recommended by the NCEP ATPIII guidelines (Table 2) mean value of LDL-C was higher and apoB was slightly higher than the recommended cut off values.

There was no significant difference between the mean values of apoB concentration in males $(102 \pm 37$ $\mathrm{mg} / \mathrm{dL})$ and females $(104 \pm 45 \mathrm{mg} / \mathrm{dL})$. Furthermore, $55.7 \%$ of the population had recommended optimal apoB value which is less than $100 \mathrm{mg} / \mathrm{dL}$.

Nearly three quarter of the population had more than $100 \mathrm{mg} / \mathrm{dL}$ (cut off value by the NCEP ATP III) for LDL-C. Furthermore, within this healthy population 38.2\% had more than $200 \mathrm{mg} / \mathrm{dL}$ of TC. All the lipid parameters positively correlated with apoB concentration whereas HDL-C had a negative correlation $(r=-0.165)$. HDL-C significantly $(\mathrm{p}<0.05)$ decreased with increase in apoB whereas LDL-C, TC/HDL-C and non-HDL-C significantly $(\mathrm{p}<0.05)$ increased with an increase in apoB concentration (Table 3$)$. 
Table 1. Socio-demographic data.

\begin{tabular}{cc}
\hline Socio-demographic data & Prevalence (\%) \\
Age & 50.6 \\
21 - 40 years & 38.2 \\
41 - 60 years & 11.2 \\
60 - 70 years & \\
Area of residence & 69.4 \\
Urban & 30.6 \\
Sub-urban & \\
Level of education ${ }^{\#}$ & 12.4 \\
Primary & 52.4 \\
Secondary & 35.3 \\
Tertiary & \\
\hline
\end{tabular}

"Primary: Grade 1 - 5, Secondary: 6-A/L, Tertiary: Graduates.

Table 2. Mean values of lipid parameters with standard deviation.

\begin{tabular}{ccc}
\hline Lipid parameters & Mean value \pm SD & Normal reference values \\
\hline TC $(\mathrm{mg} / \mathrm{dL})$ & $192 \pm 45$ & $<200 \mathrm{mg} / \mathrm{dL}$ \\
HDL $(\mathrm{mg} / \mathrm{dL})$ & $50 \pm 14$ & $>40 \mathrm{mg} / \mathrm{dL}$ \\
LDL $(\mathrm{mg} / \mathrm{dL})$ & $120 \pm 46$ & $<100 \mathrm{mg} / \mathrm{dL}$ \\
TG $(\mathrm{mg} / \mathrm{dL})$ & $123 \pm 61$ & $<150 \mathrm{mg} / \mathrm{dL}$ \\
Non-HDL-C (mg/dL) & $143 \pm 49$ & $<130 \mathrm{mg} / \mathrm{dL}$ \\
TC/HDL & $4.23 \pm 1.92$ & $<4.0$ \\
LDL/HDL & $2.72 \pm 1.67$ & $<2.5$ \\
TG/HDL & $2.52 \pm 1.89$ & $>4$ \\
ApoB (mg/dL) & $103 \pm 42$ & $<100 \mathrm{mg} / \mathrm{dL}$ \\
\hline
\end{tabular}

Table 3. Results of the Pearson correlation between the traditional lipid parameters and apoB.

\begin{tabular}{cccc}
\hline Lipid parameters & Correlation & r value & p value \\
\hline TC & Positive & 0.12 & 0.11 \\
HDL & Negative & -0.17 & $0.03^{\mathrm{a}}$ \\
LDL & Positive & 0.15 & $0.04^{\mathrm{a}}$ \\
TG & Positive & 0.01 & 0.89 \\
Non-HDL-C & Positive & 0.17 & $0.03^{\mathrm{a}}$ \\
LDL/HDL & Positive & 0.14 & 0.06 \\
TC/HDL & Positive & 0.15 & $0.04^{\mathrm{a}}$ \\
TG/HDL & Positive & 0.12 & 0.11 \\
\hline
\end{tabular}

${ }^{\mathrm{a}}$ Statistically significant. 


\subsection{Association of apoB with Other Lipid Parameters}

Participants with normal apoB concentration $(<100 \mathrm{mg} / \mathrm{dL}$ ) had lower mean values for all the lipid parameters except HDL-C when compared to the participants having high apoB concentration $(>100 \mathrm{mg} / \mathrm{dL})$. Whereas LDL-C, non-HDL-C, TC/HDL-C and TG/HDL-C were significantly $(p<0.05)$ higher in subjects with high apoB concentration (Table 4).

\section{Discussion}

A cluster of CVD risk factors prevail among South Asians, hence they are more prone to be affected with CVD than rest of the population worldwide [3]. It has also been proven that prevalence of CVD is higher in this region. Studies conducted among this population had also indicated that this variation is attributed mainly due to genetic inheritance while a genetic influence on apoB level [14] has been revealed as well. In addition, the pattern and prevalence of dyslipidaemia varied among this population [13]. Most of the studies have stated that urban residents have a higher risk of development of CVD than residents of other areas. This is attributed mainly to their westernized diet pattern, physical inactivity, and the sedentary life styles in addition to having comparatively high monthly income (>50\%) [15]. Similarly, in our study as most of the participants were from urban and suburban areas (Table 1) in spite of not being diagnosed with dyslipidaemia, $75 \%$ of the population had elevated LDL-C levels.

In the traditional lipid profile, five indices (TC, LDL-C, HDL-C, TG and TC/HDL-C) are assessed to evaluate lipid related risk of CVD [16]. Further, some studies indicate that LDL-C/HDL-C ratio or TC/HDL-C ratio acts as better indicators than lipoprotein itself in predicting CVD [17]. Even though ATP III of NCEP has recommended LDL-C as a primary target and non-HDL-C as the secondary target for the treatment of dyslipidaemia in order to prevent CVD [7] while being widely used in the practice as well, most of the recent studies have proven that apoB is the better marker for dyslipidaemia and a better predictor for the risk of CVD. Furthermore, several studies have recommended apoB as a better predictor to assess CVD risk than any other lipid parameter. The AMORIS and the INTERHEART studies, the two largest prospective studies have proven that apoB is a strong predictor of CVD [18].

This study also validates the necessity of measuring apo B as a pre-alarming single marker even before the onset of dyslipidaemia as it significantly correlates and associates with other traditional parameters (Table 3 and Table 4). Hence apoB can be used as the best predictor of CVD than any other traditional lipid parameter.

Even though non-HDL-C is being used as a predictor of CVD, recent studies have shown that apoB could be used instead of non-HDL-C. In addition apoB had a higher correlation in both males and females than the other traditional lipid parameters [9]. This study also proves that apoB in the present study population (Sri Lankan subjects) had a significant correlation and association (Table 4) with non-HDL-C suggesting apoB as a good marker to assess atherogenic lipid profile in apparently healthy subjects as well.

Table 4. Independent t-Test for lipid parameters and apoB.

\begin{tabular}{|c|c|c|c|c|}
\hline \multirow{2}{*}{ Parameter } & \multicolumn{2}{|c|}{ Mean value $\pm \mathrm{SD} \mathrm{mg} / \mathrm{dL}$} & \multirow{2}{*}{$\mathrm{p}$ value } & \multirow{2}{*}{$\mathrm{t}$ value } \\
\hline & Normal $^{*}$ & High $^{*}$ & & \\
\hline TC & $187 \pm 47$ & $199 \pm 43$ & 0.08 & 1.74 \\
\hline HDL & $53 \pm 15$ & $47 \pm 12$ & $0.00^{a}$ & 2.89 \\
\hline LDL & $114 \pm 49$ & $128 \pm 42$ & $0.04^{a}$ & 2.01 \\
\hline TG & $121 \pm 66$ & $126 \pm 55$ & 0.55 & 0.59 \\
\hline Non-HDL-C & $134 \pm 50$ & $152 \pm 46$ & $0.02^{\mathrm{a}}$ & 2.43 \\
\hline LDL/HDL & $2.50 \pm 1.80$ & $2.98 \pm 1.46$ & 0.05 & 1.91 \\
\hline TC/HDL & $3.93 \pm 1.98$ & $4.60 \pm 1.80$ & $0.02^{\mathrm{a}}$ & 2.29 \\
\hline TG/HDL & $2.17 \pm 1.46$ & $2.95 \pm 2.25$ & $0.007^{\mathrm{a}}$ & 2.71 \\
\hline
\end{tabular}

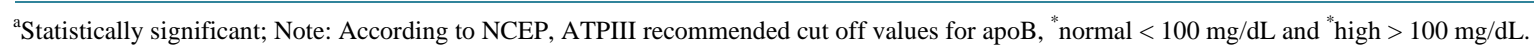


A study carried out in normal subjects as well as severe CVD group also had proven that apoB is the best marker in both groups. Different types of statistical analysis methods have also shown that apoB concentration is a good predictor in both normal and CVD subjects without any adjustments for non-lipid variables [19].

Some studies have proven that non-HDL-C has a better correlation with apoB than LDL-C thus indicating apoB as surrogate for other parameters [9]. Further current studies have also proven that apoB is the better predictor for future CVD with better diagnostic sensitivity and specificity making it a better and pertinent parameter to measure atherogenic dyslipidaemia.

LDL-C measurement is carried out by using the Friedewald formula, hence fasting is a must. This is because LDL-C depends on the measurement of fasting triglycerides. ApoB is a patient friendly measurement, as apoB measurement does not require fasting. Even though there are two types of apoB, contribution of apoB48 is less. Serum apoB is targeted mainly for apoB100 and epitopes for antibodies used in immunoassays have been raised against apoB100 [8].

Mean values of LDL-C and TG/HDL-C were higher than the optimal recommended value in this healthy population. But mean value of apoB was near the optimal value (Table 2). A study conducted in normal healthy population has found that the optimal mean value for apoB concentration is $95.2 \pm 28 \mathrm{mg} / \mathrm{dL}$ [19].

A study conducted in Sri Lanka to assess the impact of apoB and apolipoprotein-A1 in metabolic syndrome among diabetic patients indicates that increased levels of apoB is an additional CVD risk factor that clusters with other components of metabolic syndrome among diabetic patients. The mean value of apoB concentration in this Sri Lankan diabetic population was $122 \mathrm{mg} / \mathrm{dL}$ and apoB concentration significantly increased with increasing number of metabolic syndrome components [20].

A study conducted among diabetic patients who were on statin treatment had a mean apoB concentration of $650 \pm 180 \mathrm{mg} / \mathrm{dL}$ whereas the LDL-C and TG were $<40 \mathrm{mg} / \mathrm{dL}$ and $98 \mathrm{mg} / \mathrm{dL}$ respectively [8]. Therefore, measurement of LDL-C concentration only is nullified in diabetic patients who are vulnerable to have CVD. Further, there aren't any other validated procedures to assess the elevated LDL-C in diabetic subjects. Determination of apoB is comparatively low cost than all the traditional lipid parameters. In addition it has in existence a WHO approved standard which makes it easier to compare values even from different laboratories [11].

\section{Conclusions}

This study findings show that apoB has a high correlation and association with the parameters of conventional lipid profile and with the lipid parameters that are recommended as markers for dyslipidaemia in apparently healthy people. In addition, apoB can be used as a single sole marker rather than multiple traditional markers of lipid profile to determine dyslipidaemia making it economically cheaper and patient-friendly as fasting is not required.

The limitation of this study is that this study had convenience sample with cross-sectional study design.

\section{Acknowledgements}

University grant ASP/06/RE/MED/2012/21 is gratefully acknowledged.

\section{Declaration of Conflicts Interest}

There are no conflicts of interest.

\section{Financial Support}

Study was funded by University grant ASP/06/RE/MED/2012/21.

\section{References}

[1] World Health Organization (2013) Cardiovascular Diseases Fact Sheet $N^{\circ} 317$. http://www.who.int/mediacentre/factsheets/fs317/en/

[2] Ministry of Healthcare and Nutrition (2009) The National Policy for Prevention and Control of Chronic Non Communicable Diseases. Ministry of Healthcare and Nutrition, Colombo, Sri Lanka.

[3] Katulanda, P., Ranasinghe, P., Jayawardana, R., Sheriff, R. and Mathews, D.R. (2012) Metabolic Syndrome among Sri Lankan Adults: Prevalence, Patterns and Correlates. Diabetology \& Metabolic Syndrome, 4, 1-10. 
http://dx.doi.org/10.1186/1758-5996-4-24

[4] Misra, A., Luthra, K. and Vikram, N.K. (2004) Dyslipidemia in Asian Indians: Determinants and Significance. Journal of the Association of Physicians India, 52, 137-143.

[5] Enas, E.A., Chacko, V., Pazhoor, S.G., Chennikkara, H. and Devarapalli, H.P. (2007) Dyslipidemia in South Asians Patients. Current Atherosclerosis Report, 9, 367-374. http://dx.doi.org/10.1007/s11883-007-0047-y

[6] Herath, H.R.I.S., Katulanda, P., Mathews, D.R., Sheriff, M.H.R., Constantine, G.R. and De Vas Gunawardena, A.N.P., et al. (2010) Prevalence and Patterns of Dyslipidaemia among Adult Sri Lankan. $123^{\text {rd }}$ Annual Academic Session, Sri Lanka Medical Association, Colombo, 15 June 2010.

[7] Adult Treatment Panel III (2002) Third Report of the National Cholesterol Education Program (NCEP) Expert Panel on Detection, Evaluation, and Treatment of High Blood Cholesterol in Adults (Adult Treatment Panel III) Final Report. Circulation, 106, 3143-3421.

[8] Durrington, P.N. (2002) Can Measurement of Apolipoprotein B Replace the Lipid Profile in the Follow-Up of Patients with Lipoprotein Disorders? Clinical Chemistry, 48, 401-402.

[9] Levinson, S.S. (2007) Comparison of Apolipoprotein B and Non-High-Density Lipoprotein Cholesterol for Identifying Coronary Artery Disease Risk Based on Receiver Operating Curve Analysis. American Journal of Clinical Pathology, 127, 449-455. http://dx.doi.org/10.1007/s11883-007-0047-y

[10] Poonguzhali, D.V., Vinodhini, V.M., William, W. and Kumar, J.S. (2013) Evaluation of Apolipoprotein-B Levels in Dysglycemia. Asian Journal of Pharmaceuticals and Clinical Research, 6, 112-114.

[11] Harpers, C.R. and Jacobson, T.A. (2010) Using Apolipoprotein B to Manage Dyslipidemic Patients: Time for a Change? Mayo Clinic Proceedings, 85, 440-445. http://dx.doi.org/10.4065/mcp.2009.0517

[12] Nair, N. (2015) Should We Measure Apolipoproteins to Evaluate Coronary Heart Disease Risk? The NYU Langone Online Journal of Medicine. http://www.clinicalcorrelations.org/?p=5748

[13] Zhang, L., Qiao, Q. and Dong, Y.H. (2012) Ethnic Difference in Lipid Profiles. In: Kelishadi, R., Ed., DyslipidemiaFrom Prevention to Treatment, InTech. http://www.intechopen.com/books/dyslipidemia-from-prevention-to-treatment/ethnic-differences-in-lipid-profiles

[14] Mukherjee, M., Srivastava, A., Kesari, A. and Mital, B. (2005) Analysis of VNTR Loci, ApoB 3’HVR and DIS80 in North Indians. Indian Journal of Biotechnology, 4, 358-362.

[15] Tennakoon, S.U.B., Kumar, B.N., Nugegoda, D.B. and Meyer, H.E. (2010) Comparison of Cardiovascular Risk Factors between Sri Lankans Living in Kandy and Oslo. BMC Public Health, 10, 1-8. http://dx.doi.org/10.1186/1471-2458-10-654

[16] Miremadi, S., Sniderman, A. and Frohlich, J. (2010) Can Measurement of Serum Apolipoprotein B Replace the Lipid Profile Monitoring Patients with Lipoprotein Disorders? Clinical Chemistry, 48, 484-488.

[17] Vishwanath, P., Prashant, A., Acharya, A., Suma, M.N., Kiran, H.S. and Karthik, G. (2012) Use of Anthropometric Indices as Simple Predictors of Deranged Lipid Profile and at Risk Population for Future Cardiovascular Events. International Journal of Health\& Allied Science, 1, 8-12. http://dx.doi.org/10.4103/2278-344X.96411

[18] Wallidus, G. and Jungner, I. (2005) Rationale for Using Apolipoprotein B and Apolipoprotein A-I as Indicators of Cardiac Risk and as Targets for Lipid-Lowering Therapy. European Heart Journal, 26, 210-212. http://dx.doi.org/10.1093/eurheartj/ehi077

[19] Siniawski, D.A., Masson, W., Sorroche, P., Casanas, L., Krauss, J. and Cagide, A. (2010) Correlation between Apolipoprotein B to Apolipoprotein A1 Ratio and Total to HDL Cholesterol Ratio in a Healthy Population. Should Castelli Index Be Updated? Revista Argeninat de Cardioogia, 79, 33-38.

[20] Katulanda, G.W., Katulanda, P., Sheriff, M.H.R., Matthews, D.R. and Brian, S. (2009) Comparison of Apolipoproteins $\mathrm{B}$ and A1 in People with versus without Metabolic Syndrome. $122^{\text {nd }}$ Anniversary Academic Session, Sri Lanka Medical Association, Colombo, 32 March 2009. 